American Journal of Infectious Diseases 4 (2): 96-103, 2008

ISSN 1553-6203

(C) 2008 Science Publications

\title{
Ultrastructural Evidences of Hepatitis B Infection in Human Liver Biopsies Disclose Complex Assembly and Morphogenesis Pathways for Hepatitis B Virus
}

\author{
${ }^{1}$ Viviana Falcon, ${ }^{1}$ Ivon Menéndez, ${ }^{1}$ Nelson Acosta-Rivero, \\ ${ }^{4}$ Mineko Shibayama, ${ }^{1}$ Marı-C de la Rosa, ${ }^{4}$ Jose Luna- Munoz, \\ ${ }^{4}$ Magdalena Miranda-Sanchez, ${ }^{1}$ Jorge V. Gavilondo, ${ }^{1}$ Deliana Lopez, \\ ${ }^{1}$ Santiago Duenas-Carrera, ${ }^{2}$ Bienvenido Gra, ${ }^{1}$ Glay Chinea, ${ }^{2}$ Luisa Tamayo Garcia, \\ ${ }^{3}$ Waldo Garcia, ${ }^{2}$ Eduardo Vidal, ${ }^{2}$ Enrique Arus-Soler, ${ }^{1}$ Jose Silva, ${ }^{1}$ Felix Alvarez, \\ ${ }^{5}$ Emilio F. Acosta, ${ }^{1}$ Jesus Seoane, ${ }^{1}$ Juan Morales-Grillo, ${ }^{1}$ Eduardo Penton, \\ ${ }^{4}$ Juan Kouri and ${ }^{4}$ Victor Tsutsumi \\ ${ }^{1}$ Centro de Ingeniería Genética y Biotecnología, P.O. Box 6162, C.P. 10600, La Habana, Cuba \\ ${ }^{2}$ Institute of Gastroenterology, C.P. 10400, La Havana, Cuba \\ ${ }^{3}$ General Hospital "Calixto Garcia', C.P. 10400,La Havana, Cuba \\ ${ }^{4}$ Centro de Investigaciones y Estudios Avanzados (CINVESTAV-IPN), México DF, Mexico \\ ${ }^{5}$ Escuela Latinoamericana de Ciencias Médicas, La Habana, Cuba
}

\begin{abstract}
Despite of recent advances on acknowledge of hepatitis B virus (HBV) structure and biology, little is known about the morphogenesis and release of virions. In this study, the ultrastructural analysis of HBV components in liver biopsies from chronically HBV-infected patients disclosed complex assembly and morphogenesis pathways for $\mathrm{HBV}$. $\mathrm{HBV}$ core $(\mathrm{HBcAg})$ and surface (HBsAg) antigens were specifically identified in all liver biopsies from HVB-infected patients. HBcAg containing core-like particles $24-28 \mathrm{~nm}$ in diameter were observed both in nucleus and cytoplasm of hepatocytes. Dane's-like particles were detected either budding to or into the lumen of ER close to HBsAg containing tubular structures. Besides, Dane's-like particles were detected in different vesicular compartments resembling multivesicular endosomes. Other kind of enveloped HBV-like particles similar to the previously described cobra-shaped and horn-shaped particles were also observed in hepatocytes. Some of these particles were connected to the vesicle membrane through a stalk 20-22 $\mathrm{nm}$ in diameter. On the other hand, spherical subviral particles (SVP) were frequently observed in cytoplasmic vesicles. Moreover, a minor proportion of enveloped HBV-like particles budding through the plasma membrane to the extracellular space and bile canaliculi were detected. Interestingly, Dane's-like particles in the bile canaliculi and entering into ductular-like cells were shown. Strikingly, Dane's-like particles close to tubular structures and specific immunolabeling for $\mathrm{HBcAg}$ both in cytoplasm and nuclei of stellate-like cells were detected. Remarkably, HVB-like particles appeared entering hepatocytes from large cytoplasmic processes of fibrocytes raising the interesting possibility of a cell to cell passage of HBV through direct transcellular migration.
\end{abstract}

Key words: Hepatitis B virus, HBV morphogenesis, Dane's particles, electron microscopy

\section{INTRODUCTION}

Hepatitis B viruses (HBVs; hepadnaviruses), are small, enveloped viruses and a causative agent of acute and chronic viral hepatitis ${ }^{[1,2]}$. They replicate by transcription of RNA pregenomes and reverse transcription to produce the DNA genomes that are found in the virus particles secreted from infected hepatocytes ${ }^{[3]}$. Hepatitis B virus (HBV) virions exhibit unique electron-microscopical features. The complete virus, called the Dane's particle, is $42-47 \mathrm{~nm}$ in diameter ${ }^{[1]}$. The nucleocapsid, or core particle, which is composed of single core protein species $(\mathrm{HBcAg}$ ), contains a double-stranded DNA genome and the covalently attached viral polymerase and is surrounded by a membrane shell with two or three viral envelope

Corresponding Author: Viviana Falcon, Center for Genetic Engineering and Biotechnology (CIGB), P.O. Box 6162, Havana 10600, Cuba 
proteins embedded ${ }^{[4]}$. The protein envelope present on HBV surface consists of three protein types: Small (S), Medium (M) and Large (L) proteins. The $\mathrm{S}$ protein (HBsAg) represents the main component of viral envelope and of subviral particles (SVP ${ }^{[4,5]}$. SVP are seen in the form of spherical or elongated tubular structures of various lengths and 20-22 nm in diameter ${ }^{[4,5]}$. At the beginning, HBsAg is synthesized as an integral transmembrane Endoplasmic Reticulum (ER) protein, budding to the lumen in the form of particles, which undergo secretion ${ }^{[4]}$.

Dane's particles have been described in liver biopsies from HBV-infected patients ${ }^{[6-10]}$. They were similar to those visualized in human sera ${ }^{[1,11]}$. SVP have also been observed in great excess over virions. Moreover, other ultrastructural forms of HBV named tadpole- or cobra-shaped HBV with a head measuring $42 \mathrm{~nm}$ and a tail with $22 \mathrm{~nm}$ in diameter and hornshaped HBV with a head and two branches of different length have also been reported ${ }^{[1,11-13]}$.

Despite of recent advances on acknowledge of HBV structure and biology, little is known about the morphogenesis and release of virions ${ }^{[14]}$. Among factors complicating study of these processes, HBV replicates at a low rate and, therefore, the amount of HBV antigens in the liver is small, rendering its detection difficult $^{[4,15]}$. Thus, it has been shown that the direct observation of HBV budding by electron microscopy is very difficult to achieve ${ }^{[16]}$. However, electron microscopy studies might be powerful tools to elucidate some of the mechanisms involved in HBV replication and virion morphogenesis as well as HBV pathogenesis $^{[17]}$. To address these issues and to better understand virus-host interactions in $\mathrm{HBV}$ replication, an ultrastructural analysis of HBV components in liver biopsies from chronically $\mathrm{HBV}$-infected patients was examined in this study.

\section{MATERIALS AND METHODS}

Patients and samples: Patients with chronic HBV infection hospitalized for hepatitis in the Institute of Gastroenterology, Havana, Cuba were recruited after informed consent in writing was obtained. Liver needle biopsies samples were taken at the time of routine diagnostic biopsy from all patients. Seven chronically HBV-infected patients ( 3 females and 4 males, aged 2040 years) were selected based upon they were serologically positive to HBV enzyme immunoassays (Tecnosuma International, Havana, Cuba). They also were histologically confirmed as bearing chronic hepatitis and had abnormal serum alanine aminotransferase levels for at least six month before the biopsy was performed. None were seropositive for markers of hepatitis $\mathrm{C}$ virus, hepatitis $\mathrm{A}$ virus and human immunodeficiency virus by enzyme immunoassays (Tecnosuma International, Havana, Cuba). In addition, liver needle biopsies samples were taken from two HBV-uninfected healthy donor livers for transplantation purpose and from hepatitis Cinfected patients as negative controls.

Antibodies: The following mouse monoclonal antibodies (mAbs) were used: anti-HBcAg CBSSHepB.1 mAb specific for HBcAg and anti-HBsAg CBSS-HepB.2 mAb specific for HBsAg ${ }^{[17]}$.

Transmission electron microscopy: The hepatic tissue samples were fixed for $1 \mathrm{~h}$ at $4^{\circ} \mathrm{C}$ in $1 \%(\mathrm{v} / \mathrm{v})$ glutaraldehyde and $4 \%(\mathrm{v} / \mathrm{v})$ paraformaldehyde, rinsed in $0.1 \mathrm{M}$ sodium cacodylate ( $\mathrm{pH} 7.4$ ), post-fixed for $1 \mathrm{~h}$ at $4^{\circ} \mathrm{C}$ in $1 \%$ OsO4 and dehydrated in increasing concentrations of ethanol. The embedding was done as previously described ${ }^{[18]}$. Samples were examined with a JEOL/JEM 2000 EX transmission electron microscope (JEOL, Japan).

Immunoelectron microscopy (IEM): Samples of hepatic tissue were fixed with $4 \%(\mathrm{v} / \mathrm{v})$ paraformaldehyde containing $0.2 \%(\mathrm{v} / \mathrm{v})$ glutaraldehyde in $0.1 \mathrm{M}$ phosphate buffer $(\mathrm{pH} 7.3)$ at $4^{\circ} \mathrm{C}$ for $3 \mathrm{~h}$ and washed with $0.1 \mathrm{M}$ phosphate buffer $\mathrm{pH}$ 7.3. Fixed cells were dehydrated as described above, embedded in Lowicryl and polymerized by exposure to ultraviolet light at Room Temperature (RT) for $72 \mathrm{~h}$. Ultrathin sections of liver biopsies were incubated with either CBSS-HepB.1 or CBSS-HepB. 2 mAbs in phosphate buffer, for $45 \mathrm{~min}$ at RT. The sections were rinsed three times for $30 \mathrm{~min}$ at RT with $0.1 \%$ bovine serum albumin in phosphate-buffered saline pH 7.3 (BSAPBS) and incubated for $1 \mathrm{~h}$ at RT with gold-labeled (15 $\mathrm{nm}$ ) anti-mouse IgG (Amersham, UK) diluted 1:100 in BSA-PBS. As control the primary antibody was substituted by normal mouse serum. All sections were stained and analyzed with a transmission electron microscope as mentioned above.

Immunofluorescence staining: Samples were immediately fixed with $4 \%$ paraformaldehyde in PBS at $4^{\circ} \mathrm{C}$ and then mounted on gelatine-coated glass slides and stored for 2 days at $-200^{\circ} \mathrm{C}$. Later on, mounted samples were hydrated for $10 \mathrm{~min}$ in PBS and incubated with $0.2 \%$ Triton $\mathrm{X}$ in PBS during 10 min. To block non-specific antibody reaction, best results were obtained by incubating the sections with $0.2 \%$ BSA (free of IgG) (Sigma Chemical Co. St. Louis, 
Mo.USA), for 10 min at RT. After two washes in PBS$\mathrm{T}$, samples were incubated overnight at $4^{\circ} \mathrm{C}$ with either CBSS-HepB.1 or CBSS-HepB.2 mAbs (dilutions 1:20 in PBS-T). Incubations were followed by washes with PBS-T. The second incubations were accomplished with FITC-conjugated anti-mouse IgG (dilutions 1:60 in PBS-T, Vector laboratories, Inc., Burlingame, CA., USA) for $1 \mathrm{~h}$ at RT. After three washes with PBS-T the sections from all samples were counterstained with propidium iodide (dilution 1:1000, Vector laboratories, Inc. Burlingame CA., USA), followed by extensive washing in PBS-T. Immunostained samples were coverslipped in Vectashield mountaing medium (Vector Laboratories, Inc. Burlingame, CA., USA), sealed with nail polish and viewed on a confocal laser scanning microscope. Negative controls were performed by substituting the primary antibodies with normal mouse serum.

Laser confocal scanning imaging and serial section collection: Samples were viewed with a 60x (NA 1.4) objective on a Nikon microscope with attached laser confocal scanning system MRC 600 (BioRad, Watfod). Ten to twelve fields were imaged from each sample. Four to fifteen serial optical z-sections $(0.2-0.5 \mu \mathrm{m}$ thick) were collected from each observed field using the dual channel imaging with green exciter filter $(554 \mathrm{~nm})$ for propidium iodide excitation (rhodamine chanel) and the blue exciter filter $(494 \mathrm{~nm})$ for the fluorescein channel. Each series of confocal optical sections was scanned through a total of $25 \mu \mathrm{m}$. The resulting optical sections were fully projected onto two-dimensional planes using the imaging processing system of the microscope (Camos package).

\section{RESULTS}

Reaction products suggestive of HBV antigens within hepatocytes were observed in liver biopsies from the seven HBV-infected patients (Fig. 1a,b). No signals were observed in the liver biopsy specimens from the control subjects (Fig. 1c). The specific stain reaction was blocked completely by incubation with normal mouse serum as primary antibodies (not shown). Hepatocytes containing such reaction products were distributed sporadically or focally in some lobuli. Samples immunolabeled with anti-HBsAg mAbs showed perinuclear and cytoplasmic reactions (Fig. 1b). On the other hand, HBcAg was immunolabeled in both the cytoplasm and nucleus (Fig. 1a).

In five out of seven liver biopsies showing positive reactions for $\mathrm{HBV}$ antigens, the presence of $\mathrm{HBcAg}$ within cell nuclei of infected hepatocytes in the form of

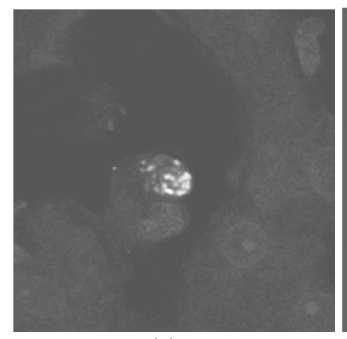

(a)

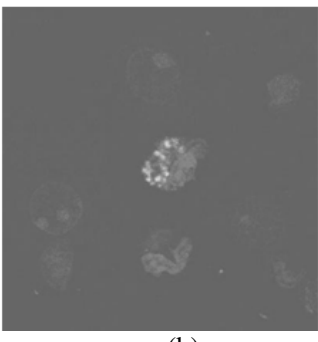

(b)

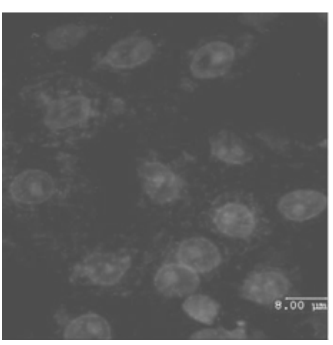

(c)

Fig. 1: Immunofluorescence staining with either antiHBcAg mAbs (a): Or anti-HBsAg mAbs (b): In liver biopsy specimens from $\mathrm{HBV}$-infected patients. (c): Liver biopsy from a healthy subject, no immunostaining was observed using any of these mAbs. $(B a r=8 \mu \mathrm{m})$

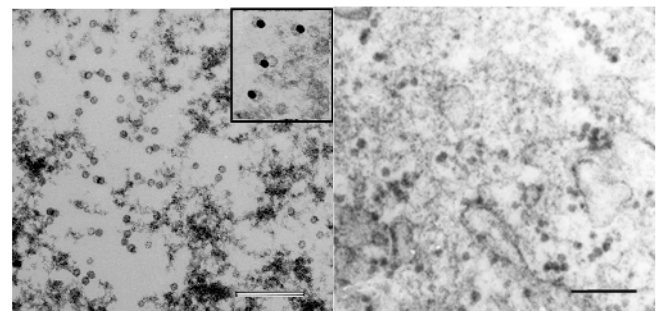

(a)

(b)

Fig. 2:Electron micrographs of core-like particles either in the nuclei or cytoplasm of hepatocytes. Fragment of either hepatocyte nuclei (a): Or cytoplasm (b): From a liver biopsy specimen of a chronically HBV-infected patient showing abundant core-like particles of 24-28 nm in diameter. Inset in a (Magnification): Immunolabeling of $\mathrm{HBcAg}$ in the nuclei of hepatocytes by IEM using anti-HBcAg mAbs. $($ Bar $=200 \mathrm{~nm})$

ring-like structures (core-like particles) of 2-28 nm in diameter were demonstrated by IEM (Fig. 2a). Most of the nuclear core-like particles were likely empty inside. However, electron-dense core-like particles (probably containing viral genome) were observed in the cytoplasm of these samples (Fig. 2b). They form groups or are spread all over the cell. 


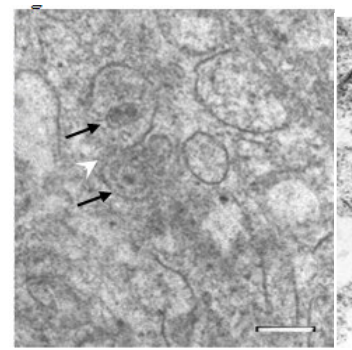

(a)

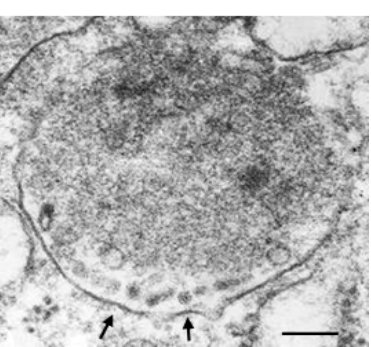

(b)
Fig. 3:Electron micrographs of Dane's-like particles in liver biopsies from HBV-infected patients. (a): Part of a hepatic cell showing vesicles containing Dane's-like Particles of 42-47 nm of diameter (black arrows) into larger cytoplasmic vesicle with a membrane invagination (white arrowhead). (b): Cytoplasmic vesicle resembling a multivesicular body containing Dane's-like particles of 42-47 nm of diameter. $($ Bar $=200 \mathrm{~nm})$

In liver biopsies showing intranuclear core-like particles, enveloped HBV-like particles resembling Dane's-like particles with diameters of 42-47 nm were observed into different cytoplasmic vesicles of hepatocytes (Fig. 3a, black arrows). Interestingly, some of these HBV-like particles containing vesicles were inside larger vesicles showing membrane invaginations (white arrowhead). Dane's-like particles also appeared in vesicles resembling Multivesicular Bodies (MVB) (Fig. 3b).

In addition, the cytoplasm of hepatocytes from these liver biopsies contained tubular structures 20-25 $\mathrm{nm}$ in diameter (Fig. 4a, white arrows). Some cells were packed with these structures; in others they were only present in part of the cytoplasm. However, these structures were not detected in liver biopsies from either healthy subjects or from $\mathrm{HCV}$-infected patients (not shown). Tubular structures were specifically immunolabeled with anti-HBsAg mAbs (Fig. 4b). No immunogold staining was observed in sections incubated with normal mouse immunoglobulins as primary antibodies (not shown). Besides, Dane's-like particles with diameters ranging from $45-55 \mathrm{~nm}$ were localized close to the tubular structures (Fig. 4a, black arrows). They appeared budding into the lumen of ER. Moreover, spherical SVP with $20-22 \mathrm{~nm}$ in diameter were frequently observed inside cytoplasmic vesicles close to tubular structures (Fig. 4a, Lower Inset: black arrows).

Other kinds of enveloped HBV-like particles were detected closed to the tubular structures. Dane's-like particles with a long tail $20-25 \mathrm{~nm}$ in diameter and a length of 230-270 $\mathrm{nm}$ resembling the previously

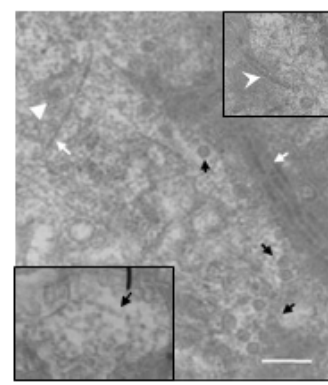

(a)

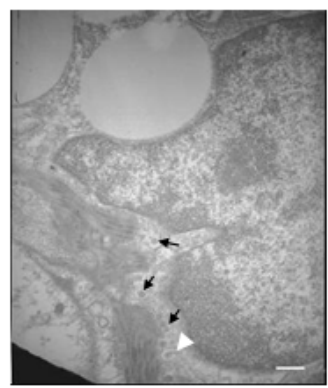

(c)

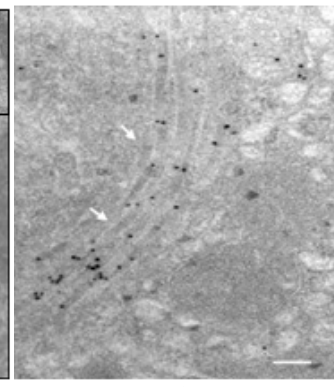

(b)

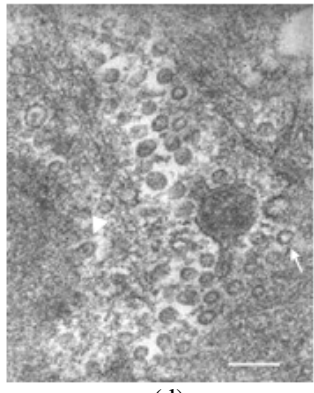

(d)
Fig. 4: Electron micrographs showing tubular structures, spherical subviral particles and Dane's-like particles in liver biopsies from HBV-infected patients. (a): Part of a hepatic cell showing tubular structures (white arrows) and different types of enveloped virus-like particles of $45-55 \mathrm{~nm}$ of diameter. Note Dane'slike particles (black arrows) budding into cisternae of ER and Cobra-shape particles with a long tail (white arrowhead). Upper Inset: Horn-like particle with two tails (white arrowhead). Lower Inset: Cytoplasmic vesicle containing spherical subviral particles $20-22 \mathrm{~nm}$ in diameter (black arrow). (b): Immunolabeling of HBsAg in the tubular structures by IEM using anti-HBsAg mAbs. (c): Part of a stellatelike cell showing tubular structures and different types of HBV-like particles of 45-55 $\mathrm{nm}$ of diameter budding into cisternae of ER (black arrows). Note Dane's-like particles connected with the cisternal membrane through a stalk (white arrowhead). (d): HBV-like particles in the extracellular space. Note corecontaining particles budding from the plasma membrane (white arrowhead). Also note empty particles (white arrow). $(\mathrm{Bar}=200 \mathrm{~nm})$

described cobra-shape particles were observed (Fig. 4a, arrowhead). In addition, Dane's-like particles with two long tails $20-25 \mathrm{~nm}$ in diameter with a variable length of 300-400 $\mathrm{nm}$ resembling the previously described horn-shape particles were detected (Fig. 4a, Upper Inset: white arrowhead). 


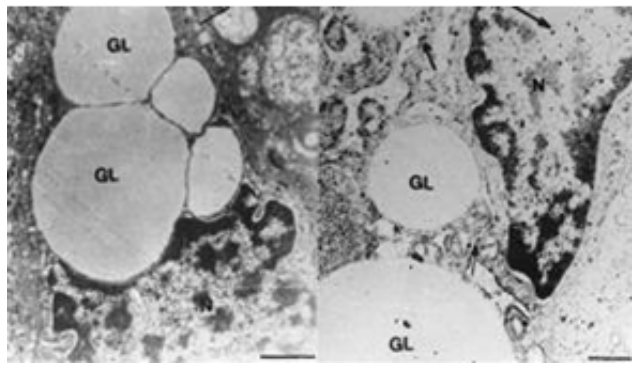

(a)

(b)

Fig. 5: Immunolabeling of HBcAg by IEM in stellatelike cells from liver biopsies of HBV-infected patients. (a): Liver biopsies from healthy subjects, no immunostaining was observed using anti-HBcAg mAbs and gold-labeled antimouse IgG. (b): Immunostaining with anti$\mathrm{HBcAg}$ revealed $\mathrm{HBcAg}$ in cytoplasm and the nuclei (black arrows). GL: Lipid droplets. $(\mathrm{Bar}=500 \mathrm{~nm})$

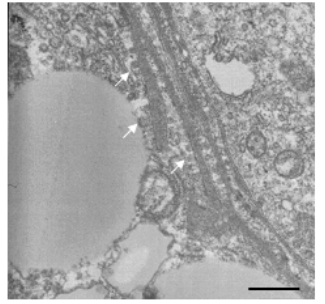

(a)

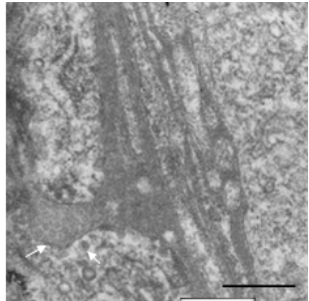

(b)

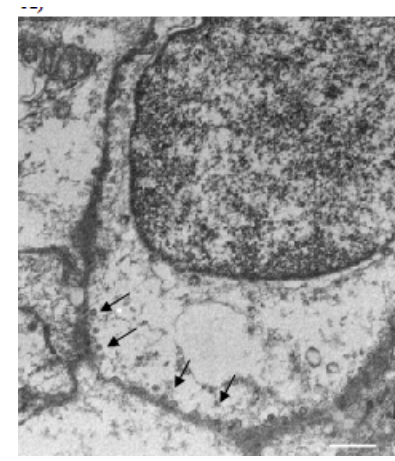

(c)

Fig. 6: Electron micrographs of liver biopsies from HBV-infected patients. (a, b): HBV-like particles protruding from large processes of fibrocytes and entering neighbour cells (white arrows). (c): HBV-like particles entering ductular-like cells (black arrows) $(\mathrm{Bar}=300$ $\mathrm{nm}$ in $\mathrm{A}$ and $\mathrm{C}, 500 \mathrm{~nm}$ in $\mathrm{B}$ )

Interestingly, tubular structures and Dane's-like particles with diameters ranging from 45-55 nm budding into the lumen of ER were also observed in stellate-like cells (Fig. 4c, black arrows). Note the presence of Dane's-like particles connected to the membrane through a stalk $20-22 \mathrm{~nm}$ in diameter (white arrowhead). Remarkably, $\mathrm{HBcAg}$ was shown to localize in stellate-like cells (Fig. 5b, arrows). Specific immunolabeling appeared distributed in the cytoplasm as well as in the nuclei. No immunogold staining was observed in liver sections from uninfected individuals (Fig. 5a) or in sections incubated with normal mouse immunoglobulins as primary antibodies (not shown).

Enveloped HBV-like particles were also localized in the extracellular space (Fig. 4d) and in bile canaliculi (not shown). Some of them appeared budding from the plasma membrane (white arrowhead). Most of these particles were clearly composed of an inner electrondense core-like particle surrounded by an envelope. However, a proportion of empty particles were observed (white arrow). In addition, these particles were not detected in hepatocytes from normal controls (not shown).

It is interesting to note that Dane's-like particles appeared protruding from large processes of fibrocytes and entering neighbour cells (Fig. 6a and b, white arrows). These cells were in close contact. Moreover, Dane's-like particles entering ductular-like cells were seen (Fig. 6c, black arrows).

\section{DISCUSSION}

Both immunofluorescence and IEM studies specifically identified HBV antigens in all liver biopsies from HVB-infected patients. In agreement with previous studies, $\mathrm{HBcAg}$ was disclosed in the cytoplasm and cell nuclei of hepatocytes ${ }^{[9,19]}$. On the other hand, HBsAg was noted in the cytoplasm as well as on tubular structures ${ }^{[7,10]}$. It is interesting to note that the HBV genome has been detected in hepatocytes from these liver biopsies using an in situ hybridization assay $^{[20]}$ followed by confocal microscopy (Falcon et al, unpublished results). Thus, data support the infection of hepatocytes by HBV in these samples.

Some ultrastructural lesions associated with chronic type $\mathrm{B}$ hepatitis are related to molecular structure of $\mathrm{HBV}^{[7,21]}$. In the present work we were able to detect core and enveloped HBV-like particles in liver biopsy samples from $\mathrm{HBV}$-infected individuals with chronic hepatitis. Core-like particles 24-28 nm in diameter were located both in nucleus and cytoplasm. Nuclear $\mathrm{HBcAg}$ possibly represents accumulation of empty nucleocapsids whereas cytoplasmic $\mathrm{HBcAg}$ may be considered as active virus replication ${ }^{[19]}$. Enveloped HBV-like particles showed a hepadnavirus-like morphology (Dane's particles) with ultrastructural 
characteristics, size and morphology similar to those previously reported in different HBV models ${ }^{[1,7]}$.

HBV virion budding and release currently are still not fully understood. Results from this study suggest complex assembly and morphogenesis pathways for HBV. Dane's-like particles were detected either budding to or into the lumen of ER close to $\mathrm{HBsAg}$ containing tubular structures. This may represent a primary site for HVB virion assembly and budding ${ }^{[6,7]}$. On the other hand, Dane's-like particles were observed into vesicles resembling MVB. Besides, vesicles containing Dane's-like particles were detected inside larger vesicles heterogeneous in size showing membrane invaginations. It is possible that these vesicles derived from multivesicular endosomes (MVE) ${ }^{[22]}$. Thus, they may represent newly forming intermediates in either the degradation pathway or the HBV virion maturation. This is in agreement with a recent report showing assembly and budding of virion particles into a novel type of intracellular vesicles using duck HBV as a model system ${ }^{[23]}$. These vesicles derived from ER having unique identity and properties of ER, intermediate compartment, endosomes and MVB.

The MVB pathway is responsible to sort cargo proteins destined for degradation, lysosomal functions, or exosomal release toward intraluminal vesicles ${ }^{[22]}$. MVB functions have been implicated in budding by different enveloped RNA viruses ${ }^{[24]}$. Most of these viruses access the MVB machinery through its virusencoded late assembly domains and bud through the plasma membrane. In addition, recent communications have reported the involvement of MVB functions in HBV virion maturation and egress using virusreplicating liver cell lines ${ }^{[25,26]}$. HBV virions have been suggested to bud into internal MVB-related compartments and exit the cell by the exosome pathway.

In this study, a minor proportion of enveloped HBV-like particles budding through the plasma membrane to the extracellular space were detected. Some of these particles consist of empty particles with no electron-dense nucleocapsid possibly representing either defective interfering particles ${ }^{[27]}$ or SVP ${ }^{[23]}$. In addition, enveloped particles with an electron-dense nucleocapsid likely corresponding to virions were observed. Some authors have shown that HBV envelope proteins do not appear in the plasma membrane ${ }^{[4]}$. So, based on the molecular characterization of the hepadnaviral morphogenesis it is assumed that hepatitis B virions are formed by budding of the capsids through an intracellular membrane ${ }^{[4]}$. However, other reports had shown that HBV envelope proteins could be immunodetected in the plasma membrane of hepatocytes from liver biopsies of HBVinfected patients ${ }^{8,19,28]}$. In addition, HBV-like particles budding from the plasma membrane of hepatocytes has been reported ${ }^{[10,29]}$. Thus, it seems that a proportion of HBV could bud through plasma membrane during chronic infection in humans. Taken together, it is possible to suggest that similar to enveloped RNA viruses that use components of the MVB sorting pathway for the formation of progeny particles that mostly bud at the plasma membrane of infected cells, HBV may utilize MVB functions for budding not only into internal MVB-related compartments but also through the plasma membrane.

On the other hand, large tubular structures (that were specifically immunostained with anti-HBsAg $\mathrm{mAbs}$ ) were detected in liver biopsy samples from HBV-infected patients. These structures were similar to those previously described in liver biopsies from HBVinfected patients ${ }^{[6,7]}$. In addition, spherical SVP were observed inside vesicles. Previous ultrastructural studies have proposed that HBV virions and SVP exploit a common morphogenetic pathway ${ }^{[5]}$. Nevertheless, results from this work showed that Dane's-like particles and SVP localize in different cellular compartments. This is in agreement with recent evidences showing that the viral and subviral HBV assembly pathways seemingly differ in their requirement for cell functions and trafficking routes ${ }^{[25,26]}$. While HBV virions budding required the involvement of MVB functions, the mechanisms of SVP production showed to be clearly distinct and likely independent of MVB functions ${ }^{[25,26]}$.

Strikingly, Dane's-like particles were also observed close to tubular structures in stellate-like cells. In addition, stellate-like cells were specifically immunolabeled with anti-HBcAg both in cytoplasm and nuclei suggesting that HBV could replicate in this cell type. Stellate cells have a central role in the pathogenesis of chronic liver injury ${ }^{[30]}$. Our finding raises the possibility that $\mathrm{HBV}$ could directly modulate the stellate cells functions playing a role in $\mathrm{HBV}$ pathogenesis. Further studies are necessary to define this interesting topic both at cellular and molecular levels.

In addition, other kind of enveloped HBV-like particles were observed in this study. They resembled the previously described tadpole or cobra-shaped and horn-shaped particles ${ }^{[1,11-13]}$. A recent report suggested that these particles are the native replicative form of $\mathrm{HBV}^{[13]}$. However, in agreement with this study, previous reports had shown the presence of Dane's particles connected to the vesicle membrane through either a short or a long stalk $20 \mathrm{~nm}$ in diameter ${ }^{[10,31]}$. Besides, it has been shown that interfering with some of the components of the machinery that catalyzes MVB vesicle budding inhibited HIV-1 assembly and budding, resulting in the accumulation of partially budded 
virions that remain connected to the plasma membrane ${ }^{[32]}$. Therefore, another interesting hypothesis would be that these particles arise due to MBV functions impairment leading to deficient fission events during viral budding.

Our results showed Dane's-like particles in the bile canaliculi and entering into ductular-like cells suggesting the involvement of the biliary system in the replicative cycle of HVB and the pathogenesis of HVBinduced liver disease. These findings support previous observations showing HBV components in ductular cells $^{[33]}$ as well as Dane's particles in bile canaliculi and budding of core particles from hepatocytes into the bile canaliculi $^{[12,29]}$.

By using the DHBV model, it has been previously suggested that liver sinusoidal endothelial cells play a key role in the initial uptake of DHBV into the liver ${ }^{[34]}$. The authors proposed that DHVB initially scavenged by LSEC were thereafter released to infect adjacent hepatocytes. In this study, HVB-like particles appeared entering hepatocytes from large cytoplasmic processes of fibrocytes. This raises the interesting possibility that a cell to cell passage of $\mathrm{HBV}$ through direct transcellular migration may occur in vivo. A more profound study in this field could further contribute to clarify the life cycle of HBV.

\section{ACKNOWLEDMENT}

The authors thank Nilda Tamayo for their excellent technical assistance. The study was supported by the Center for Genetic Engineering and Biotechnology, Havana, Cuba.

\section{REFERENCES}

1. Dane, D.S., C.H. Cameron and M. Briggs, 1970. Virus-like particles in serum of patients with Australia-antigen-associated hepatitis. Lancet, 1: 695-698.

2. Lee, W., 1997. Hepatitis B virus infection. New Engl. J. Med., 337: 1733-1745.

3. Summers, J. and W.S. Mason, 1982. Replication of the genome of a hepatitis B-like virus by reverse transcription of an RNA intermediate. Cell, 29: 403-415.

4. Bruss, V., 2004. Envelopment of the hepatitis B virus nucleocapsid. Virus Res., 106: 199-209.

5. Patient, R., C. Hourioux, P.Y. Sizaret, S. Trassard, C. Sureau and P. Roingeard, 2007. Hepatitis B virus subviral envelope particle morphogenesis and intracellular trafficking, J. Virol., 81: 3842-3851.
6. Stein, O., M. Fainaru and Y. Stein, 1972. Visualization of virus-like Particles in endoplasmic reticulum of hepatocytes of Australia antigen carriers. Lab. Invest., 26: 262-269.

7. Gerber, M.A., S. Hadziyannis, C. Vissoulis, F. Schaffner, F. Paronetto and H. Popper, 1974. Electron microscopy and immunoelectronmicroscopy of cytoplasmic hepatitis B antigen in hepatocytes. Am. J. Pathol., 75: 489-502.

8. Yamada, G. and P.K. Nakane, 1977. Hepatitis B core and surface antigens in liver tissue. Light and electron microscopic localisation by the peroxidase-labelled antibody method. Lab. Invest., 36: 649-59.

9. Cabral, G.A., 1978. Immunohistochemical and electron microscopic detection of hepatits B surface and core antigens. Exp. Mol. Pathol., 29: 156-169.

10. Yamada, G., Y. Sakamoto, M. Mizuno, T. Nishihara, T. Kobayashi, T. Takahashi and H. Nagashima, 1982. Electron and immmunoelectron microscopic study of Dane particle formation in chronic hepatitis B virus infection. Gastroenterology, 83: 348-356.

11. Stannard, L.M., J. Moodie, J.A. Keen and A. Kipps, 1973. Electron microscopic study of the distribution of the Australia antigen in individual sera of 50 serologically positive blood donors and two patients with serum hepatitis. J. Clin. Path., 26: 209-216.

12. Yamada, G., Y. Sakamoto, M. Mizuno, T. Kobayashi and H. Nagashima, 1980. Hepatitis B virus associated particles in the bile canaliculus. Acta Medica Okayama, 34: 143-146.

13. Kaito, M., H. Ohba, J. Chiba, M. Kohara, H. Tanaka, N. Fujita, E.C. Gabazza, S. Watanabe, M. Konishi and Y. Adachi, 2006. The ultrastructural morphology of native hepatitis B virus. Med. Mol. Morphol., 39: 136-145.

14. Dryden, K.A., S.F. Wieland, C. Whitten-Bauer, J.L. Gerin, F.V. Chisari and M. Yeager, 2006. Native Hepatitis B Virions and Capsids Visualized by Electron Cryomicroscopy. Molecular Cell, 22: 843-850.

15. Nowak, M.A., S. Bonhoeffer, A.M. Hill, R. Boehme, H.C. Thomas and H. McDade, 1996. Viral dynamics in hepatitis B virus infection, Proc. Natl. Acad. Sci. USA., 93: 4398-4402.

16. Roingeard, P. and C. Sureau, 1998. Ultrastructural analysis of hepatitis B virus in HepG2-transfected cells with special emphasis on subviral filament morphogenesis. Hepatology, 28: 1128-1133. 
17. Falcon, V., N. Baranosky; F.O. Castro, C. Montero, M. González, O. Hayes, O. Ancheta, B. Gra and S. Mandado, 1993. Ultrastructural and immunocytochemical characteristics of hepatocytes from hepatitis B virus infected chimpanzees. Tissue Cell, 25: 865-873.

18. Falcon, V., N. Acosta-Rivero, G. Chinea, J. Gavilondo, M.C. de la Rosa, I. Menéndez, S. Dueñas-Carrera, A. Viña, W. García, B. Gra, M. Noa, E. Reytor, M.T. Barcelo, F. Álvarez and J. Morales-Grillo, (2003). Ultrastructural evidences of $\mathrm{HCV}$ infection in hepatocytes of chronically $\mathrm{HCV}$-infected patients, Biochem. Biophys. Res. Commun., 305: 1085-1090.

19. Gowans, E.J. and C.J. Burrell, 1985. Widespread presence of cytoplasmic $\mathrm{HBcAg}$ in hepatitis B infected liver detected by improved immunochemical methods, J. Clin. Pathol., 38: 393-398.

20. Falcon, V., N. Acosta-Rivero, M. Shibayama, J. Luna-Muñoz, M. Miranda-Sánchez, J. Gavilondo, M.C. de la Rosa, I. Menéndez, B. Gra, W. García, S. Dueñas-Carrera, J. Silva, G. Chinea, M. González-Bravo, F. Álvarez, J. Morales, J. Kouri and V. Tsutsumi, 2005. Detection of HCV components and pathological reactions in apoptotic hepatocytes from chronically $\mathrm{HCV}$-infected patients. Am. J. Inf. Dis., 1: 12-24.

21. Meuleman, P., L. Libbrecht, S. Wieland, R. De Vos, N. Habib, A. Kramvis, T. Roskams and G. Leroux-Roels, 2006. Immune Suppression Uncovers Endogenous Cytopathic Effects of the Hepatitis B Virus. J. Virol., 80: 2797-2807.

22. Gruenberg, J. and H. Stenmark, 2004. The biogenesis of multivesicular endosomes. Nat. Rev., 5: 317-323.

23. Mhamdi, M., A. Funk, H. Hohenberg, H. Will and H. Sirma, 2007. Assembly and Budding of a Hepatitis B Virus Is Mediated by a Novel Type of Intracellular Vesicles. Hepatology, 46: 95-106.

24. P.D. Bieniasz, 2006. Late budding domains and host proteins in enveloped virus release. Virology, 344: 55-63.

25. Lambert, C., T. Döring and R. Orange, 2007. Hepatitis B Virus Maturation is Sensitive to Functional Inhibition of ESCRT-III, Vps4 and $\gamma 2-$ Adaptin. J. Virol., 81: 9050-9060.
26. Watanabe, T., E.M. Sorensen, A. Naito, M. Schott, S. Kim and P. Ahlquist, 2007. Involvement of host cellular multivesicular body functions in hepatitis B virus budding, Proc. Natl. Acad. Sci. USA, 104: 10205-10210.

27. Yuan, T.T-T., M-H. Lin, D-S. Chen and C. Shih, 1998. A Defective Interference-Like Phenomenon of Human Hepatitis B Virus in Chronic Carriers. J. Virol., 72: 578-584.

28. Ray, M.B., V.J. Desmet, J. Fevery, J. De Groote, A.F. Bradburne and J. Desmyter, 1976. Distribution patterns of hepatitis B surface antigen (HBsAg) in the liver of hepatitis patients, J. Clin. Path., 29: 94-100.

29. Yamada, G., Y. Sakamoto, M. Mizuno, T. Kobayashi and H. Nagashima, 1980. Electron microscopic observation of hepatitis B virus budding from hepatocytes into bile canaliculi, Acta Medica Okayama, 34: 203-207.

30. Gressner, A.M. and R. Weiskirchen, 2006. Modern pathogenetic concepts of liver fibrosis suggest stellate cells and TGF-beta as major players and therapeutic targets. J. Cell Mol. Med., 10: 76-99.

31. Gerber, M.A., M.A. Sells, M.L. Chen, S.N. Thung, S.S. Tabibzadeh, A. Hood and G. Acs, 1988. Morphologic, immunohistochemical and ultrastructural studies of the production of hepatitis B virus in vitro. Lab. Invest., 59: 173-180.

32. Strack, B., A. Calistri, S. Craig, E. Popova and H.G. Gottlinger, 2003. AIP1/ALIX is a binding partner for HIV-1 p6 and EIAV p9 functioning in virus budding. Cell, 114: 689-699.

33. Delladetsima, J.K., I. Vafiadis, N.C. Tassopoulos, V. Kyriakou, A. Apostolaki and T. Smyrnoff, 1994. HBcAg and HBsAg expression in ductular cells in chronic hepatitis B. Liver, 14: 71-75.

34. Breiner, K.M., H. Schaller and P.A. Knolle, 2001. Endothelial cell-mediated uptake of a hepatitis B virus: a new concept of liver targeting of hepatotropic microorganisms, Hepatology, 34: 803-808. 